\title{
Genome-wide association study confirms extant PD risk loci among the Dutch
}

\author{
Javier Simón-Sánchez ${ }^{\star, 1}$, Jacobus J van Hilten ${ }^{2}$, Bart van de Warrenburg ${ }^{3}$, Bart Post ${ }^{3}$, Henk W Berendse ${ }^{4}$, \\ Sampath Arepalli ${ }^{5}$, Dena G Hernandez ${ }^{5}$, Rob MA de Bie ${ }^{6}$, Daan Velseboer ${ }^{6}$, Hans Scheffer ${ }^{7}$, Bas Bloem ${ }^{3}$, \\ Karin D van Dijk ${ }^{4}$, Fernando Rivadeneira ${ }^{8,9}$, Albert Hofman ${ }^{8}$, André G Uitterlinden ${ }^{8,9}$, Patrizia Rizzu ${ }^{1}$, \\ Zoltan Bochdanovits ${ }^{1}$, Andrew B Singleton ${ }^{5}$ and Peter Heutink ${ }^{1}$
}

In view of the population-specific heterogeneity in reported genetic risk factors for Parkinson's disease (PD), we conducted a genome-wide association study (GWAS) in a large sample of PD cases and controls from the Netherlands. After quality control (QC), a total of 514799 SNPs genotyped in 772 PD cases and 2024 controls were included in our analyses. Direct replication of SNPs within SNCA and BST1 confirmed these two genes to be associated with PD in the Netherlands (SNCA, rs2736990: $P=1.63 \times 10^{-5}, \mathrm{OR}=1.325$ and $\left.B S T 1, \mathrm{rs} 12502586: P=1.63 \times 10^{-3}, \mathrm{OR}=1.337\right)$. Within $S N C A$, two independent signals in two different linkage disequilibrium (LD) blocks in the $3^{\prime}$ and $5^{\prime}$ ends of the gene were detected. Besides, post-hoc analysis confirmed GAK/DGKQ, HLA and MAPT as PD risk loci among the Dutch (GAK/DGKQ, rs2242235: $P=1.22 \times 10^{-4}, \mathrm{OR}=1.51$; $H L A$, rs4248166: $P=4.39 \times 10^{-5}, \mathrm{OR}=1.36$; and $M A P T$, rs3785880: $P=1.9 \times 10^{-3}, \mathrm{OR}=1.19$ ).

European Journal of Human Genetics (2011) 19, 655-661; doi:10.1038/ejhg.2010.254; published online 19 January 2011

Keywords: SNCA; BST1; GAK/DGKQ; HLA; MAPT; PD

\section{INTRODUCTION}

A genetic contribution to Parkinson's disease (PD) is well recognized ${ }^{1-3}$ with $\sim 10 \%$ of the cases carrying mutations that lead to rare Mendelian forms of the disease. ${ }^{4}$ Recently, independent genomewide association studies (GWAS) established an unequivocal role for common genetic variants in the etiology of $\mathrm{PD}^{5-11}$ and suggested a population-specific genetic heterogeneity, with SNCA, PARK16, $B S T 1$ and LRRK2 as shared risk loci for $P D,{ }^{6-8,10,11}$ and MAPT as an European-specific risk locus. ${ }^{6,7}$ Besides, variation in the GAK/DGKQ, ${ }^{5}$ the HLA region ${ }^{10}$ and a locus in chromosome $12 \mathrm{q} 24^{11}$ has been proposed to exert a risk for PD in different European populations.

To determine the role of these loci in the Dutch population and to find new genetic factors exerting a risk for PD, we carried out what is, to our knowledge, the first GWAS for PD in the Dutch population.

\section{SUBJECTS AND METHODS}

Subjects

As a product of a national collaborative venture, a total of 841 PD patients were recruited from four different centers within the Netherlands (Scales for Outcomes in Parkinson's disease, SCOPA, http://www.scopa-propark.eu; the Academic Medical Center Amsterdam, AMC, http://www.amc.uva.nl); the Parkinson Centrum Nijmegen, ParC, http://www.umcn.nl; and the VU University medical centre, VUmc, http://www.vumc.nl). All patients were self-reported Caucasian individuals from the Netherlands. The assessed samples consisted of 533 males and 308 females with a mean age at onset ranging from 16 to 84 years $($ mean $=57.5$ years; standard deviation=12). For more information about these samples, please visit the websites listed above.

Genome-wide genotyping data from 2082 control participants from the Rotterdam study III ${ }^{12-14}$ (ERGO Young) genotyped with Human610K beadchips from Illumina (http://www.illumina.com) were used as our control population. Of these, 912 were males and 1116 females. The mean age was 53.75 years with a range of $45-95$ years.

\section{Genotyping}

All 841 PD cases were genotyped at 592839 unique positions with Human660W-Quad beadchips from Illumina, a powerful tool for GWAS. For more information about this genotyping platform, please visit http:// www.illumina.com.

Quality control (QC) procedures

After extensive QC approaches (see Supplementary Material for details), the final number of fully genotyped samples from the Netherlands was 2796 including 772 cases and 2024 controls. Each of these was genotyped in a total of 514799 unique autosomal SNPs.

\section{Statistical analyses}

Quanto software was used to estimate power (University of South California, http://hydra.usc.edu/gxe). Odds ratios (OR) considering a $P$-value of $9.71 \times 10^{-8}$ (genome-wide significance level after Bonferroni correction) and different allele frequencies were calculated (Supplementary Figure 1).

${ }^{1}$ Department of Clinical Genetics, Section of Medical Genomics, VU University Medical Centre, Amsterdam, The Netherlands; ${ }^{2}$ Department of Neurology, Leiden University Medical Center, Leiden, The Netherlands; ${ }^{3}$ Department of Neurology, Radboud University Nijmegen Medical Centre, Nijmegen, The Netherlands; ${ }^{4}$ Department of Neurology, VU University Medical Centre, Amsterdam, The Netherlands; ${ }^{5}$ Laboratory of Neurogenetics, National Institute on Aging, National Institutes of Health, Bethesda, MD, USA; ${ }^{6}$ Department of Neurology, Academic Medical Center, University of Amsterdam, Amsterdam, The Netherlands; ${ }^{7}$ Department of Human Genetics, Radboud University Nijmegen Medical Centre, Nijmegen, The Netherlands; ${ }^{8}$ Department of Epidemiology, Erasmus University Medical Center, Rotterdam, The Netherlands and ${ }^{9}$ Department of Internal Medicine, Erasmus Medical Center, Rotterdam, The Netherlands

*Correspondence: Dr J Simón-Sánchez, Department of Clinical Genetics, Section of Medical Genomics, VU University Medical Centre, Amsterdam, North Holland 1081 BT, The Netherlands. Tel: +31 20598 3662; Fax: +31 20598 3596; E-mail: j.simonsanchez@vumc.nl

Received 28 July 2010; revised 30 November 2010; accepted 14 December 2010; published online 19 January 2011 
For association analyses, all estimates and tests were carried out with PLINK $^{15}$ (http://pngu.mgh.harvard.edu/ purcell/plink/).

For each SNP that successfully passed our QC process, a multi-covariate logistic regression analysis following an additive model was applied. Covariates used in our model were gender, age at onset and age at ascertainment (for cases and controls, respectively), and the first two components of the MDS values after calculation of pairwise IBS. Although the genomic inflation factor (based on median $\left.\chi^{2}\right)$ was low $(\lambda=1.06$, Supplementary Figure 3 ), we chose to adjust for genomic control to correct for possible population substructure.

In a first approach aiming to determine (in the Dutch) the role of previously identified PD genetic risk factors, we decided to look closely at the results for SNCA (chromosome 4q21), ${ }^{5-7}$ MAPT locus (chromosome 17q21.1), ${ }^{5,7}$ LRRK2 (chromosome 12q12), ${ }^{6,7}$ PARK16 ${ }^{6,7}$ (chromosome 1q32), BST1 (chromosome $4 \mathrm{p} 15),{ }^{6}$ the GAK/DGKQ locus (chromosome 4p16), ${ }^{5}$ the HLA region (chromosome $6 \mathrm{p} 21.3)^{10}$ and chromosome 12q24 locus. ${ }^{11}$ A total of 30 SNPs from these loci were selected for closer scrutiny.

The LD structure of the associated loci was analyzed using Haploview $4.1^{16}$ (Broad Institute of MIT and Harvard, www.broad.mit.edu/mpg/haploview/) and blocks delimited using the D'-based confidence interval method developed by Gabriel et al $^{17}$ as implemented in Haploview. Risk haplotypes were counted and OR values were plotted with R v.2.7.2 (http://cran.r-project.org/).

A logistic regression analysis of the most associated SNP in each block conditioned to variation at the most associated SNP in the other block (if applicable) was carried out to test for statistical independence of the signals detected.

For $S N C A$, the population attributable risk (PAR) was calculated using the formula $\mathrm{PAR}=(p(\mathrm{RR}-1)) /(p(\mathrm{RR}-1)+1) \times 100$, where $p$ is the prevalence of the risk allele in the population and RR is the relative risk. As we showed that association from the $3^{\prime}$ and $5^{\prime} \mathrm{LD}$ block is independent (see results), the combined PAR was calculated with the formula $\mathrm{CPAR}=$ $1-\left(1-\mathrm{PAR}_{3^{\prime} \text { block }}\right) \times\left(1-\mathrm{PAR}_{5^{\prime} \text { block }}\right)$.

For MAPT locus, the presence of alleles in the $\mathrm{H} 1$ and $\mathrm{H} 2$ haplotypes was accomplished using rs1981997 as a haplotype tag SNP because the major $(\mathrm{G})$ and minor (A) alleles of this SNP are fixed in the $\mathrm{H} 1$ and $\mathrm{H} 2$ haplotypes, respectively. ${ }^{9,18}$ To determine which of the associated alleles in the MAPT locus were present in $\mathrm{H} 1$ (previously associated with $\mathrm{PD}^{19-24}$ ) a two-locus haplotype association analysis of rs1981997 and SNPs in the MAPT region was carried out using PLINK. ${ }^{15}$

Although we are aware that the sample size of this cohort has a limited power and a GWAS would probably fail to find any associated locus after correcting for 514799 independent tests, we decided to carry out this analysis to look for specific PD risk loci in the Dutch population. For this purpose, each genotyped SNP was tested for association using the multi-covariate logistic regression explained above.

\section{RESULTS}

After QC, a total of 772 Dutch PD cases and 2024 controls from the Rotterdam study ${ }^{12-14}$ genotyped in a total of 514799 unique autosomal SNPs, were included in our analyses (Table 1). To assess the homogeneity of our cohort, identity by state (IBS) distances were calculated. This analysis revealed that both cases and controls share common ancestry (Supplementary Figure 1A) consistent with that seen in CEU (CEPH Europeans from Utah) (Supplementary Figure $1 \mathrm{~B}, \mathrm{C})$. Power calculations showed that our sample has adequate power to detect variants conferring a risk with an OR of 1.4-1.5 depending on the minor allele frequency of such variant (Supplementary Figure 2).
As we are aware that our population is of limited size to detect risk loci with the effect size previously described for PD in Europeans at the significance level requested by GWAS, the first objective of this project was to determine the role, in the Dutch population, of previously identified genetic risk factors. Thus, association results from 30 SNPs in SNCA, ${ }^{5-7}$ MAPT locus, ${ }^{5,7}$ LRRK2, ${ }^{6,7}$ PARK16, ${ }^{6}$ $B S T 1^{6}$ and GAK/DGKQ, ${ }^{5,10} H L A^{10}$ and chromosome $12 \mathrm{q} 24$ loci, ${ }^{11}$ were selected for close scrutiny. Two SNPs in SNCA and BST1 were significantly associated with $\mathrm{PD}$ in our population after correcting for 30 independent tests $(P<0.00166$ in both instances, Table 2). Although no association was found in any of the other SNPs, a trend toward an association in the DGKQ/GAK and MAPT loci was detected (Table 2). Post-hoc analysis of results at these two locations and the HLA region, provided evidence of the role of these three loci in the pathogenesis of PD in the Dutch. No association was found in LRRK2, PARK16, or the chromosome 12q24 locus. A more detailed description of the results obtained at each locus is given below.

\section{SNCA}

Although association of SNCA with PD is well established, it remains unclear whether it is derived from the $3^{\prime}$ or the $5^{\prime}$ end of the gene. ${ }^{6-8,25-32}$ In an effort to delineate the signal at this locus, we examined the LD structure across this region. This analysis revealed two LD blocks delimited at the intron 4 of SNCA as previously described $^{6-8,25}$ (Supplementary Figure 4). Although the strongest signal detected in SNCA was located at the $3^{\prime}$ block (rs2736990, $P=1.63 \times 10^{-5}$ ), a closer examination of the association signals revealed that SNPs both in the $3^{\prime}$ (lowest $P=1.63 \times 10^{-5}$, rs 2736990 ) and the $5^{\prime}$ blocks (lowest $P=1.78 \times 10^{-3}$, rs356188) of the gene appeared to be associated with PD in our population (Supplementary Table 1). A logistic regression analysis using the allele dosage of the most associated SNP in each block as a covariate, did not change the results observed at the most associated signal in the other, indicating that the two signals are independent (Supplementary Table 2) and being in disagreement with previous results. ${ }^{7,25,32} \mathrm{LD}$ analysis between these two SNPs revealed a $r^{2}$ value of 0.028 and a D' value of 0.388 , further supporting this hypothesis.

Examination of the risk conferred by each of the haplotypes contained in these blocks confirmed these results and showed that a single haplotype contained in the $3^{\prime}$ block and two other in the $5^{\prime}$ block exerted the largest risk for PD in our population with ORs of 1.42, 1.41 and 1.48, respectively (Supplementary Figure 5).

\section{BST1}

Rs12502586 showed significant association with PD in our population, with a $P$-value of $1.63 \times 10^{-3}$ and an OR of 1.337 . A closer look into the association results at this locus revealed that three SNPs presented with $P$-values of $\sim 10^{-3}$ (range $1.63 \times 10^{-3}-3.33 \times 10^{-3}$, Supplementary Table 3 ). Haplotype analysis of this region showed the existence of two LD blocks containing haplotypes highly associated with PD ( $P$-values of $6 \times 10^{-4}$ and $4 \times 10^{-4}$, respectively). These blocks span from $L O C 285550$ to intron 3 of BST1 and from intron 4 of BST1 to intron 8 of this same gene. The associated haplotypes exert a risk for

Table 1 Population characteristics of the Dutch cohort used for these experiments

\begin{tabular}{|c|c|c|c|c|c|}
\hline & Country of origin & Number & Male/female ratio & Age at onset (Mean (range)) & Age at sampling (Mean (range)) \\
\hline Cases & The Netherlands & 772 & 1.75 & $55.3(16-84)$ & $62.36(29-89)$ \\
\hline RSIII Controls & The Netherlands & 2024 & 0.78 & - & $53.75(45-95)$ \\
\hline
\end{tabular}


Table 2 Replication of previous GWAS results in the Dutch cohort

\begin{tabular}{|c|c|c|c|c|c|c|c|}
\hline \multirow[b]{2}{*}{$S N P$} & \multirow[b]{2}{*}{ Chromosome } & \multirow[b]{2}{*}{ Position } & \multirow[b]{2}{*}{ Locus } & \multicolumn{2}{|c|}{ Previous GWAS } & \multicolumn{2}{|c|}{ Dutch GWAS } \\
\hline & & & & $\mathrm{P}$-value & $O R$ & $\mathrm{P}$-value & $O R$ \\
\hline rs11931074 & $4 q 22$ & 90858538 & SNCA & $1.62 \times 10^{-14}$ & 1.46 & $2.15 \times 10^{-3}$ & 1.387 \\
\hline rs3857059 & $4 q 22$ & 90894261 & SNCA & $3.74 \times 10^{-15}$ & 1.48 & $2.13 \times 10^{-3}$ & 1.387 \\
\hline rs2736990 & $4 q 22$ & 90897564 & SNCA & $2.24 \times 10^{-16}$ & 1.23 & $1.63 \times 10^{-5}$ & 1.325 \\
\hline rs393152 & $17 q 21$ & 41074926 & MAPT & $1.95 \times 10^{-16}$ & 0.77 & $9.45 \times 10^{-3}$ & 0.8167 \\
\hline rs17563986 & $17 q 21$ & 41347100 & MAPT & $1.67 \times 10^{-14}$ & 0.78 & $7.65 \times 10^{-3}$ & 0.8119 \\
\hline rs1981997 & $17 q 21$ & 41412603 & MAPT & $3.55 \times 10^{-14}$ & 0.78 & $7.69 \times 10^{-3}$ & 0.812 \\
\hline rs8070723 & $17 q 21$ & 41436901 & MAPT & $9.64 \times 10^{-14}$ & 0.79 & $8.64 \times 10^{-3}$ & 0.8146 \\
\hline rs2532274 & $17 q 21$ & 41602941 & MAPT & $8.24 \times 10^{-11}$ & 0.83 & $9.16 \times 10^{-3}$ & 0.8161 \\
\hline rs2532269 & $17 q 21$ & 41605885 & MAPT & $5.62 \times 10^{-13}$ & 0.79 & 0.014 & 0.8255 \\
\hline rs2668692 & $17 q 21$ & 41648797 & MAPT & $7.87 \times 10^{-14}$ & 0.79 & 0.02 & 0.8338 \\
\hline rs169201 & $17 q 21$ & 42145386 & MAPT & $4.39 \times 10^{-14}$ & 0.78 & 0.056 & 0.8617 \\
\hline rs199533 & $17 q 21$ & 42184098 & MAPT & $1.09 \times 10^{-14}$ & 0.78 & 0.050 & 0.8581 \\
\hline rs11564162 & $12 q 12$ & 38729159 & LRRK2 & $9.52 \times 10^{-5}$ & 0.87 & 0.961 & 0.9962 \\
\hline rs2896905 & $12 q 12$ & 38779683 & LRRK2 & $7.81 \times 10^{-3}$ & 0.93 & 0.909 & 0.9926 \\
\hline rs1491923 & $12 q 12$ & 38877384 & LRRK2 & $1.55 \times 10^{-5}$ & 1.14 & 0.705 & 1.026 \\
\hline rs947211 & 1q32 & 202484322 & PARK16 & 0.01 & 0.93 & 0.123 & 0.8892 \\
\hline rs708730 & $1 q 32$ & 202509437 & PARK16 & $1.59 \times 10^{-3}$ & 0.9 & 0.848 & 1.017 \\
\hline rs823128 & $1 q 32$ & 203980001 & PARK16 & $7.29 \times 10^{-8}$ & 0.66 & 0.385 & 0.8316 \\
\hline rs823156 & $1 q 32$ & 204031263 & PARK16 & $7.60 \times 10^{-4}$ & 0.89 & 0.838 & 0.9827 \\
\hline rs11240572 & 1q32 & 204074636 & PARK16 & $6.11 \times 10^{-7}$ & 0.67 & 0.961 & 1.01 \\
\hline rs11931532 & $4 p 15.3$ & 15334864 & BST1 & 0.44 & 0.94 & 0.642 & 1.095 \\
\hline rs12502586 & $4 p 15.3$ & 15335662 & BST1 & 0.07 & 1.08 & $1.63 \times 10^{-3}$ & 1.337 \\
\hline rs12645693 & $4 p 15.3$ & 15338632 & BST1 & 0.44 & 0.94 & 0.766 & 1.06 \\
\hline rs4698412 & $4 p 15.3$ & 15346446 & BST1 & 0.03 & 0.94 & 0.021 & 0.8616 \\
\hline rs4538475 & $4 p 15.3$ & 15347035 & BST1 & 0.27 & 0.98 & 0.602 & 0.9563 \\
\hline rs12646913 & $4 p 15.3$ & 15348374 & BST1 & 0.03 & 0.92 & 0.372 & 0.8931 \\
\hline rs1564282 & $4 p 16$ & 842313 & DGKQ/GAK & $6.7 \times 10^{-7}$ & 1.7 & $4.56 \times 10^{-3}$ & 1.309 \\
\hline rs11248060 & $4 p 16$ & 954359 & DGKQ/GAK & $2.5 \times 10^{-7}$ & 1.69 & 0.013 & 1.27 \\
\hline rs3129882 & $6 p 21.3$ & 32517508 & HLA & $2.9 \times 10^{-8}$ & 1.31 & 0.312 & 1.07 \\
\hline rs4964469 & $12 q 24$ & 105474117 & $12 q 24$ & $2.38 \times 10^{-7}$ & 1.16 & 0.381 & 1.04 \\
\hline
\end{tabular}

Abbreviations: GWAS, genome-wide association study; SNP, single nucleotide polymorphism.

A total of 30 SNPs significantly associated with PD in four GWAS performed in European ${ }^{5,7,10,11}$ and Japanese ${ }^{6}$ populations were selected for closer scrutiny. $P$-values and odds ratios for these SNPs are compared with the results at those same positions in the Dutch cohort. To note, although variation in rs3129882 was not associated with PD in our population, post-hoc analysis revealed 36 SNPs nominally associated with PD in this locus (see $H L A$ region results section).

PD with an OR of 1.24 and 1.36, respectively (Supplementary Figure 6). A logistic regression analysis of the most associated SNP in each block (rs4583752 and rs12502586, respectively) conditioned to variation at the most associated SNP in the other, yielded a prominent reduction of the detected association, indicating that both risk haplotypes are tagging the same risk variant (Supplementary Table 4). The linkage disequilibrium between these two had $r^{2}$ value of 0.280 and a D' of 0.938 , further supporting the previous results.

\section{GAK/DGKQ}

Only two out of the three SNPs in the GAK/DGKQ locus, which were reported to be associated with PD by Pankratz et al ${ }^{5}$ were genotyped in our cohort. One of these (rs1564282), which is most associated in Pankratz's study, was associated with PD in our population (Table 1). Although this association did not surpass our conservative Bonferroni correction for 30 independent tests, post-hoc analysis revealed the existence of $11 \mathrm{SNPs}$ with $P$-values $<0.05$ spanning $260.5 \mathrm{~kb}$ (lowest $P=1.22 \times 10^{-4}, \mathrm{rs} 2242235$ ) (Supplementary Table 5, Supplementary Figure 7). Due to this, and because Bonferroni correction is unnecessarily punitive when replicating genes that are known to be associated with a certain trait, we consider these results as positive.

The two most associated SNPs in this region (rs2242235 and rs 4690296 with $P$-values of $1.22 \times 10^{-4}$ and $2.02 \times 10^{-4}$, respectively) are located in PCGF3, a gene $78 \mathrm{~kb}$ away from GAK and $188 \mathrm{~kb}$ away from $D G K Q$, which encodes a protein with unknown function containing a C3HC4 type RING finger for protein-protein interaction. These two SNPs are contained in two different haplotype blocks spanning from intron 1 to intron 8 of PCGF3 and intron 8 to intron 9 of this same gene, respectively. In addition, a predicted transcript, LOC100118084 is contained in the first block. Each of the two blocks contain a single haplotype associated with $\mathrm{PD}$ in our population $\left(P=2 \times 10^{-4}\right.$ and $7.38 \times 10^{-5}$, respectively) with an OR of 1.47 and 1.52 , respectively (Supplementary Figure 8 ). The most associated SNPs in each of these haplotype blocks are in strong LD with each other $\left(r^{2}=0.818, \mathrm{D}^{\prime}=0.916\right)$, indicating that they are, most likely, tagging the same risk variant in the region. A logistic regression analysis using the allele dosage at the other marker as a covariate confirmed this hypothesis (Supplementary Table 6).

\section{$H L A$ region}

In the GWAS carried out by Hamza et al, ${ }^{10}$ only one SNP (rs3129882) in this region exceeded genome-wide significance level. After in silico replication and meta-analysis of the most significant SNPs, another six SNPs in this region were associated with PD in their population. ${ }^{10}$ Of these, only rs3129882 was present in our genotyping platform. Although this SNP was not associated with PD in our population 
$(P=0.13)$, post-hoc analysis of this region revealed the existence of 36 SNPs with $P$-values below 0.05 (lowest $P=4.39 \times 10^{-5}$, rs4248166) spanning a total of $440.1 \mathrm{~kb}$ (Supplementary Table 7, Supplementary Figure 9).

The four most associated SNPs in this region ( $P$-values ranging from $4.39 \times 10^{-5}$ to $7.98 \times 10^{-5}$ ) are located in two consecutive LD blocks. Each of these blocks contains a haplotype associated with PD in our population ( $P$-values of $6.90 \times 10^{-5}$ and $6.63 \times 10^{-5}$, respectively) with ORs of 1.32 and 1.28, respectively (Supplementary Figure 10). The most associated SNPs in each of these haplotype blocks are in strong LD with each other $\left(r^{2}=0.758, D^{\prime}=0.984\right)$, indicating that they are tagging the same risk variant. A logistic regression analysis using the allele dosage at the other marker as a covariate confirmed this hypothesis (Supplementary Table 8).

The next association signal in this locus lies on rs17533090. Although this signal is $223.46 \mathrm{~kb}$ away from the one explained in the previous paragraph, it is in relatively strong $\mathrm{LD}$ with it $\left(r^{2}=0.376, \mathrm{D}^{\prime}=0.616\right)$, indicating that they are, most likely, tagging the same risk variant. A logistic regression analysis using the allele dosage at rs4248166 as a covariate confirmed this hypothesis (Supplementary Table 9).

\section{MAPT locus}

Again, Bonferroni correction is unnecessarily conservative when replicating genes known to be associated with PD. A total of 20 SNPs in this locus were nominally associated with PD in our population (lowest $P=1.9 \times 10^{-3}$ at rs3785880) (Supplementary Table 10). Genotypes at rs1981997 allowed us to differentiate between $\mathrm{H} 1$ and $\mathrm{H} 2$ haplotypes at this locus as the major (G) and the minor (A) alleles of this SNP are fixed in the H1 and $\mathrm{H} 2$ haplotypes, respectively. ${ }^{9}, 18$ This analysis showed that the $\mathrm{H} 1$ haplotype frequency in the Dutch population is $77.64 \%$.

Two-locus haplotype analysis of this SNP and the most associated SNPs in our data set $(P<0.01)$ revealed medium to high $r^{2}$ values in all SNP pairs (Supplementary Figure 11). A two-locus association analysis showed that, in all instances, positive effect sizes were found in haplotypes in which the $G$ allele of rs1981997 was present (Supplementary Table 11).

\section{Genome-wide analysis}

As expected, none of the SNPs tested reached genome-wide significance (Figure 1). The most significant signal was found on rs7995973, with a $P$-value of $5.41 \times 10^{-6}$ and an OR of 0.72 . This SNP is part of an associated locus containing 39 SNPs nominally associated with PD in our population of which eight had a $P$-value below $10^{-3}$ (Supplementary Table 1). This locus is on chromosome 13q31 and contains LOC729479 and SPRY2 (Supplementary Figure 12).

In an attempt to replicate these results, logistic regression models were applied to these 39 SNPs from two recently published GWAS datasets in European populations. ${ }^{5,7}$ This approach failed to find any association after correcting for 39 independent tests (Supplementary Table 12).

\section{DISCUSSION}

In this study, we present the results of the first GWAS of PD in the Dutch population. Given the population-specific heterogeneity in reported genetic risk factors for $\mathrm{PD},{ }^{6,7}$ the first objective of this project was to determine the role, in our population, of the genetic risk factors identified in previous GWAS in European and Japanese populations. $^{5-7,9}$ This approach confirmed SNCA and BST1 as PD risk loci. Association of the tested SNPs in GAK/DGKQ, HLA and MAPT loci did not surpass our stringent Bonferroni correction for 30 independent tests. However, given the strong a priori for each of these loci, correcting across all the tests carried out is unnecessarily conservative. For this reason and because post-hoc analyses revealed the existence of 11, 36 and 20 SNPs nominally associated with PD at these loci (GAK/DGKQ, HLA and MAPT, respectively), we are confident that the signals detected represent a true association with PD.

Although a role of SNCA in the risk for PD is well established, it is still unclear whether this association is derived from the $3^{\prime}$ or the $5^{\prime}$ end of the gene. ${ }^{6-8,25-32}$ The data presented here supports the hypothesis that two different signals in these blocks are present in the Dutch population, which is in controversy with previous results. ${ }^{7,28,30-32}$ In a report by Mueller et al, ${ }^{25}$ the authors also detected two association signals in the $3^{\prime}$ and $5^{\prime}$ blocks of SNCA. Interestingly, when stratifying their cohort into males and females, as well as young and old PD groups (according to the median age of 57 years), additional significant association in the intron 4 region of the $5^{\prime}$ block was found for the female and the young patients' subgroups. Stratifying our data on the basis of gender and median age at onset/ascertainment did not replicate such differences between either of the subgroups (data not shown). These results do not support gender and age heterogeneity for PD at this locus in the Dutch population, but support the fact that two different variants are exerting a risk for PD in our population.

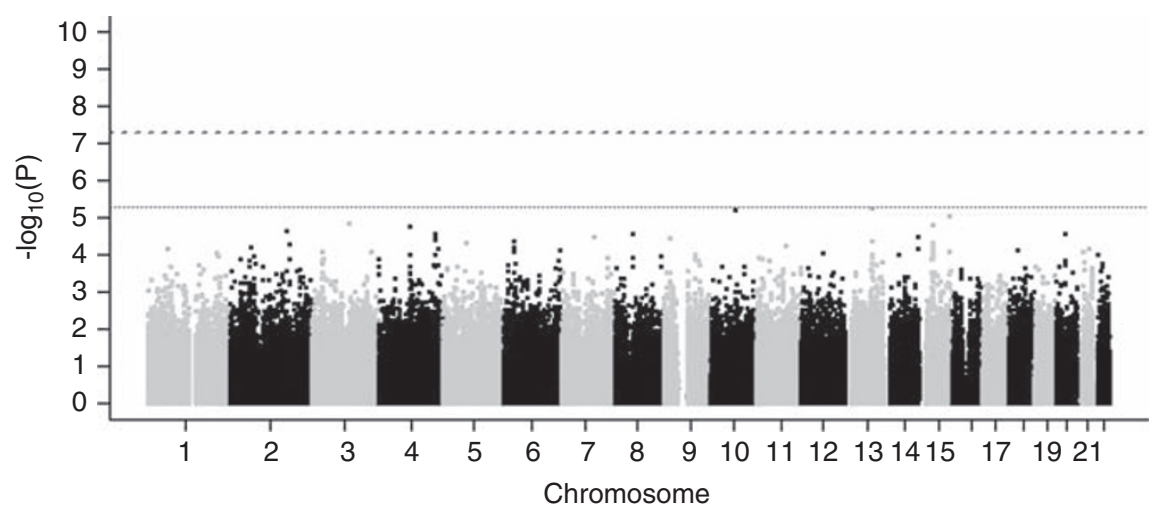

Figure 1 Manhattan plot of the results obtained after applying a logistic regression model following an additive mode of association. Age, sex and the two first components of the Multidimensional Scaling (MDS) plot were used as covariates. No SNP reached genome-wide significance level after multiple tests correction. 
Collectively, these results indicate that the population attributable risk (PAR) by variation at the SNCA locus in the Dutch is larger than previously described in other European populations, ${ }^{7}$ as it should be calculated by adding the effect of variation from each independent LD block. When the most associated SNPs at each block are considered, the combined PAR at the SNCA locus would be $17.56 \%$ (6.39\% at the $3^{\prime}$ and $11.97 \%$ at the $5^{\prime}$ block).

Several groups have focused their association studies on polymorphisms in the promoter region of SNCA, especially a microsatellite marker $\sim 10 \mathrm{~kb}$ upstream of the gene (REP1, D4S3481). Although there are some conflicting results regarding the association of this marker with $\mathrm{PD},{ }^{33-37}$ most reports consistently describe a positive association between the longer forms of REP1 and $\mathrm{PD}^{26,28,38,39}$, Gene expression differences caused by this repeat have also been described both in vitro ${ }^{35,40}$ and in vivo. ${ }^{41,42}$ We agree that examining this microsatellite in our population would help understanding whether the effect detected on the $5^{\prime}$ block is a residual effect of variation in REP1 caused by LD between the associated alleles. However, these experiments could not be performed because only genotypic information was available for the control population used in this study.

Results derived from the analyses performed herein, successfully replicate the association at BST1. Although association at this locus was originally presented as specific for the Japanese population, ${ }^{6,7}$ it has recently been replicated in British ${ }^{8}$ and French ${ }^{11}$ populations as well. Thus, this is the third study implicating BST1 on PD risk in a European population.

Although association at this locus was not detected in the GWAS carried out in a combined cohort of German and American samples, ${ }^{7}$ separating the data set by country of origin shows that rs12502586 is significantly associated with PD in the Germans, but not in North Americans ( $P$-values of $4.82 \times 10^{-3}$ and 0.179 , respectively) (Supplementary Table 13).

These results together point to the hypothesis that variation in BST1 represents a risk factor for PD in Japanese ${ }^{6}$ and European populations ${ }^{7,8,11}$ (British, French, German and Dutch populations) and that this effect has been diluted in the North America. ${ }^{7}$ A recent report by Hamza et $a l^{10}$ in which a larger cohort of North American samples have been assayed (2000 North American PD cases versus 971 typed in the previous German/North American study), identified two of the six reported SNPs at this locus to be nominally associated with PD. These results point to the hypothesis that the association of BST1 is, indeed, diluted in North America and that large datasets are needed to detect the effect of variation at this locus.

Given the known European population genetic substructure (specially between northern and southern European populations groups $)^{43,44}$ and that American population represents an admixture of individuals with northern and southern European ancestry, ${ }^{10,45}$ one would expect allele frequency differences between North American and European populations at the most heterogeneous loci. In case of rs12502586, the allele frequency of the associated allele has been diluted from 11.24 and $10.41 \%$ in the Dutch and German controls, respectively, to $9.3 \%$ in the North American controls (Supplementary Table 14). Owing to the small size effect of the associated allele, this minor allele frequency changes can lead to differences of statistical power to detect association. Thus, it is not surprising that there is a diminished power to detect association at BST1 locus in the North American population.

The events that trigger neuronal degeneration in $\mathrm{PD}$ remain unknown. Some authors argue that PD may result from inflammatory processes within the nervous system. ${ }^{46-48}$ From this perspective, it is interesting that BST1 encodes a molecule that facilitates pre-B-cell growth $^{49}$ and has an aminoacid sequence that is 33\% similar to CD38, a key player in inflammatory processes. Although no autoimmune disease has been found to be a convincing risk factor for PD, a possible pathogenic link has been proposed between rheumatoid arthritis and PD. ${ }^{50-52}$ It is noteworthy that BST1 expression is enhanced in bone marrow stromal cell lines derived from patients with rheumatoid arthritis. ${ }^{49}$

Interestingly, another region with genes implicated in neuroinflammation (HLA region) has recently been associated with PD. ${ }^{10}$ In this report, the authors identified rs3129882 to be associated with PD in their population and chose to name this locus PARK18. Although rs3129882 is not associated with PD in our population, post-hoc analysis revealed 36 SNPs nominally associated with PD in the Dutch (lowest $P=4.39 \times 10^{-5}$ ), confirming, for the first time, the role of this locus in PD etiology.

Association between certain HLA antigens and PD has largely been suggested since postmortem analysis of the substantia nigra $(\mathrm{SN})$ in PD patients revealed the presence of activated microglia. ${ }^{46}$ In addition, the frequency of various $H L A$ antigens was increased in patients with $\mathrm{PD}$ and in patients dying from postencephalitic PD. ${ }^{53-55}$ Although PD is clearly not an autoimmune disorder, the occurrence of a localized attack of microglia during the disease course has been demonstrated with epidemiological, ${ }^{56,57}$ animal model ${ }^{58,59}$ and cell culture $^{60-62}$ approaches. Zhang et al ${ }^{63}$ reported that aggregated $\alpha$-synuclein activates microglia which, as a consequence, are toxic toward cultured dopaminergic neurons.

Thus, is it hypothesized that in response to something related to $\alpha$-synuclein accumulation, microglial cells are activated to undergo a neuroinflammation process that, ultimately, kills the dopaminergic neurons of the SN.

In a recent GWAS by Pankratz and colleagues, the authors carried out a GWAS focusing only on familial PD cases. With this approach they detected evidence of association to several chromosomal regions including a locus containing GAK, TMEM175 and DGKQ, of which $G A K$ is of special interest because it was one of the 137 genes shown to be differentially expressed in PD cases in a gene expression profiling approach of parkinsonian SN. ${ }^{64}$ In our cohort, only two out of the three SNPs reported to be associated with PD by Pankratz et al, ${ }^{5}$ were successfully genotyped, one of them (rs1564282) being associated with PD in our population.

Although this association did not surpass our conservative Bonferroni correction, the a priori for this hypothesis is strong, as it has been replicated in three independent studies. ${ }^{8,10,11}$ Thus, we would at most correct for the SNPs tested within this locus and both of them would be significantly associated with $\mathrm{PD}$ in our population $(P<0.025$ in both instances, Table 2$)$.

Post-hoc analysis revealed the existence of 11 SNPs nominally associated with $\mathrm{PD}$ at this locus. Interestingly, the two most associated ones are located in PCGF3, which is $78 \mathrm{~kb}$ away from GAK and $188 \mathrm{~kb}$ away from $D G K Q$, and encodes a protein with unknown function containing a C3HC4 type RING finger for protein-protein interaction. Although the function of this transcript is still unknown, it is expressed in the brain. Haplotype analyses also point to this gene as the one carrying the risk variants for the development of PD in the Dutch population, pointing to population-specific heterogeneity for $\mathrm{PD}$ in this genomic region with different genes exerting risk in different Caucasian populations. Further genotyping of cases and controls from different populations will help understanding the role of this locus in PD.

No significant association was detected in the MAPT locus after multiple tests correction. This is probably because of the relatively 
small size of the sample used for these experiments, as this locus is a well-known PD risk locus among Europeans. ${ }^{5,7,9,23,65,66}$ If the same hypothesis as that presented for $G A K / D G K Q$ is applied, five out of the nine tested SNPs would be borderline significant (in the range of $10^{-3}$ ) supporting a role of this locus in the development of PD among the Dutch.

In our population, associated alleles at this locus occur in the H1 haplotype. Genotyping of a larger cohort of Dutch PD cases and controls will help confirming these results and defining what $\mathrm{H} 1$ subhaplotypes exert the risk for PD in the Dutch.

Last, we failed to find any new PD risk loci in the Dutch population. The most associated locus in our cohort was located in chromosome 13q31 and contained LOC729479 and SPRY2. As the signal detected was not significant after multiple test correction and because we failed to replicate it in two other Caucasian GWAS data sets, ${ }^{5,7}$ we refrained from drawing any further conclusions. For the same reason, further conclusions cannot be drawn for other clusters of nominally associated SNPs in regions with interesting biological candidates for PD that did not reach genome-wide significance level. A table showing the top 100 results is displayed in the Supplementary Material (Supplementary Table 10).

In summary, we presented the results of the first GWAS in PD in the Dutch population. These data directly confirmed SNCA and BST1 as PD genes in the Dutch population. Besides, post-hoc analysis confirmed a role for DGKQ/GAK, HLA and MAPT loci in modulating the risk for $\mathrm{PD}$ in this population.

\section{CONFLICT OF INTEREST}

The authors declare no conflict of interest.

\section{ACKNOWLEDGEMENTS}

We thank the subjects involved in this study for making this work possible. We also want to thank the Hersenstichting Nederland (http://www.hersenstichting.nl), the Neuroscience Campus Amsterdam and the section of Medical genomics (MGA) and the Intramural Research Program of the National Institute on Aging (National Institutes of Health, Department of Health and Human services; project number Z01 AG000949-04) for supporting in part the work presented here. Last, we would like to thank the Prinses Beatrix Fonds (http://www.prinsesbeatrixfonds.nl) for sponsoring this work.

1 Burn DJ, Mark MH, Playford ED et al: Parkinson's disease in twins studied with 18F-dopa and positron emission tomography. Neurology 1992; 42: 1894-1900.

2 Piccini P, Morrish PK, Turjanski N et al: Dopaminergic function in familial Parkinson's disease: a clinical and 18F-dopa positron emission tomography study. Ann Neurol 1997; 41: 222-229.

3 Piccini P, Burn DJ, Ceravolo R, Maraganore D, Brooks DJ: The role of inheritance in sporadic Parkinson's disease: evidence from a longitudinal study of dopaminergic function in twins. Ann Neurol 1999; 45: 577-582.

4 Pirkevi C, Lesage S, Brice A, Basak AN: From genes to proteins in mendelian Parkinson's disease: an overview. Anat Rec (Hoboken) 2009; 292: 1893-1901.

5 Pankratz N, Wilk JB, Latourelle JC et al: Genomewide association study for susceptibility genes contributing to familial Parkinson disease. Hum Genet 2009; 124: 593-605.

6 Satake W, Nakabayashi Y, Mizuta I et al: Genome-wide association study identifies common variants at four loci as genetic risk factors for Parkinson's disease. Nat Genet 2009; 41: 1303-1307.

7 Simon-Sanchez J, Schulte C, Bras JM et al: Genome-wide association study reveals genetic risk underlying Parkinson's disease. Nat Genet 2009; 41: 1308-1312.

8 The UK Parkinson's Disease Consortium and The Wellcome Trust Case Control Consortium 2: Dissection of the genetics of Parkinson's disease identifies an additional association $5^{\prime}$ of SNCA and multiple associated haplotypes at 7q21. Hum Mol Genet 2011; 20: 345-353.

9 Edwards TL, Scott WK, Almonte C et al: Genome-wide association study confirms SNPs in SNCA and the MAPT region as common risk factors for Parkinson disease. Ann Hum Genet 2010; 74: 97-109.
10 Hamza TH, Zabetian CP, Tenesa A et al: Common genetic variation in the HLA region is associated with late-onset sporadic Parkinson's disease. Nat Genet 2010; 42: 781-785.

11 Saad M, Lesage S, Saint-Pierre A et al: Genome-wide association study confirms BST1 and suggests a locus on 12q24 as risk loci for Parkinson's disease in the European population. Hum Mol Genet 2011; 20: 615-627.

12 Hofman A, Grobbee DE, de Jong PT, van den Ouweland FA: Determinants of disease and disability in the elderly: the Rotterdam Elderly Study. Eur J Epidemiol 1991; 7: 403-422.

13 Hofman A, Breteler MM, van Duijn CM et al: The Rotterdam Study: objectives and design update. Eur J Epidemiol 2007; 22: 819-829.

14 Hofman A, Breteler MM, van Duijn CM et al: The Rotterdam Study: 2010 objectives and design update. Eur J Epidemiol 2009; 24: 553-572.

15 Purcell S, Neale B, Todd-Brown K et al: PLINK: a tool set for whole-genome association and population-based linkage analyses. Am J Hum Genet 2007; 81: 559-575.

16 Barrett JC, Fry B, Maller J, Daly MJ: Haploview: analysis and visualization of LD and haplotype maps. Bioinformatics 2005; 21: 263-265.

17 Gabriel SB, Schaffner SF, Nguyen $\mathrm{H}$ et al: The structure of haplotype blocks in the human genome. Science 2002; 296: 2225-2229.

18 Stefansson $\mathrm{H}$, Helgason $\mathrm{A}$, Thorleifsson $\mathrm{G}$ et al: A common inversion under selection in Europeans. Nat Genet 2005; 37: 129-137.

19 Golbe LI, Lazzarini AM, Spychala JR et al: The tau AO allele in Parkinson's disease. Mov Disord 2001; 16: 442-447.

20 Maraganore DM, Hernandez DG, Singleton AB et al: Case-control study of the extended tau gene haplotype in Parkinson's disease. Ann Neurol 2001; 50: 658-661.

21 Skipper L, Wilkes K, Toft M et al: Linkage disequilibrium and association of MAPT H1 in Parkinson disease. Am J Hum Genet 2004; 75: 669-677.

22 Zhang J, Song Y, Chen H, Fan D: The tau gene haplotype h1 confers a susceptibility to Parkinson's disease. Eur Neurol 2005; 53: 15-21.

23 Zabetian CP, Hutter CM, Factor SA et al: Association analysis of MAPT H1 haplotype and subhaplotypes in Parkinson's disease. Ann Neurol 2007; 62: 137-144.

24 Tobin JE, Latourelle JC, Lew MF et al: Haplotypes and gene expression implicate the MAPT region for Parkinson disease: the GenePD Study. Neurology 2008; 71: 28-34.

25 Mueller JC, Fuchs J, Hofer $\mathrm{A}$ et al: Multiple regions of alpha-synuclein are associated with Parkinson's disease. Ann Neurol 2005; 57: 535-541.

26 Maraganore DM, de Andrade M, Elbaz A et al: Collaborative analysis of alpha-synuclein gene promoter variability and Parkinson disease. JAMA 2006; 296: 661-670.

27 Mizuta I, Satake W, Nakabayashi Y et al: Multiple candidate gene analysis identifies alpha-synuclein as a susceptibility gene for sporadic Parkinson's disease. Hum Mol Genet 2006; 15: 1151-1158.

28 Winkler S, Hagenah J, Lincoln S et al: Alpha-synuclein and Parkinson disease susceptibility. Neurology 2007; 69: 1745-1750.

29 Kay DM, Factor SA, Samii A et al: Genetic association between alpha-synuclein and idiopathic Parkinson's disease. Am J Med Genet B Neuropsychiatr Genet 2008; 147B: 1222-1230.

30 Myhre R, Toft M, Kachergus J et al: Multiple alpha-synuclein gene polymorphisms are associated with Parkinson's disease in a Norwegian population. Acta Neurol Scand 2008; 118: 320-327.

31 Rajput A, Vilarino-Guell C, Rajput ML et al: Alpha-synuclein polymorphisms are associated with Parkinson's disease in a Saskatchewan population. Mov Disord 2009; 24: 2411-2414.

32 Sotiriou S, Gibney G, Baxevanis AD, Nussbaum RL: A single nucleotide polymorphism in the 3'UTR of the SNCA gene encoding alpha-synuclein is a new potential susceptibility locus for Parkinson disease. Neurosci Lett 2009; 461: 196-201.

33 Izumi Y, Morino H, Oda M et al: Genetic studies in Parkinson's disease with an alphasynuclein/NACP gene polymorphism in Japan. Neurosci Lett 2001; 300: 125-127.

34 Khan N, Graham E, Dixon P et al: Parkinson's disease is not associated with the combined alpha-synuclein/apolipoprotein E susceptibility genotype. Ann Neurol 2001; 49: 665-668.

35 Holzmann C, Kruger R, Saecker AM et al: Polymorphisms of the alpha-synuclein promoter: expression analyses and association studies in Parkinson's disease. J Neural Transm 2003; 110: 67-76.

36 Spadafora P, Annesi G, Pasqua AA et al: NACP-REP1 polymorphism is not involved in Parkinson's disease: a case-control study in a population sample from southern Italy. Neurosci Lett 2003; 351: 75-78.

37 Tan EK, Chai A, Teo YY et al: Alpha-synuclein haplotypes implicated in risk of Parkinson's disease. Neurology 2004; 62: 128-131.

38 Farrer M, Maraganore DM, Lockhart $\mathrm{P}$ et al: alpha-Synuclein gene haplotypes are associated with Parkinson's disease. Hum Mol Genet 2001; 10: 1847-1851.

39 Pals P, Lincoln S, Manning J et al: alpha-Synuclein promoter confers susceptibility to Parkinson's disease. Ann Neurol 2004; 56: 591-595.

40 Chiba-Falek O, Nussbaum RL: Effect of allelic variation at the NACP-Rep1 repeat upstream of the alpha-synuclein gene (SNCA) on transcription in a cell culture luciferase reporter system. Hum Mol Genet 2001; 10: 3101-3109.

41 Cronin KD, Ge D, Manninger P et al: Expansion of the Parkinson disease-associated SNCA-Rep1 allele upregulates human alpha-synuclein in transgenic mouse brain. Hum Mol Genet 2009; 18: 3274-3285.

42 Linnertz C, Saucier L, Ge D et al: Genetic regulation of alpha-synuclein mRNA expression in various human brain tissues. PLoS One 2009; 4: e7480.

43 Seldin MF, Shigeta R, Villoslada $P$ et al: European population substructure: clustering of northern and southern populations. PLoS Genet 2006; 2: e143.

44 Moskvina V, Smith M, Ivanov D et al: Genetic differences between five European populations. Hum Hered 2010; 70: 141-149. 
45 Price AL, Butler J, Patterson $\mathrm{N}$ et al: Discerning the ancestry of European Americans in genetic association studies. PLoS Genet 2008; 4: e236.

46 McGeer PL, Itagaki S, Boyes BE, McGeer EG: Reactive microglia are positive for HLA-DR in the substantia nigra of Parkinson's and Alzheimer's disease brains. Neurology 1988; 38: 1285-1291.

47 Hirsch EC, Hunot S: Neuroinflammation in Parkinson's disease: a target for neuroprotection? Lancet Neurol 2009; 8: 382-397.

48 Rugbjerg K, Friis S, Ritz B, Schernhammer ES, Korbo L, Olsen JH: Autoimmune disease and risk for Parkinson disease: a population-based case-control study. Neurology 2009; 73: 1462-1468.

49 Kaisho T, Ishikawa J, Oritani $\mathrm{K}$ et al: BST-1, a surface molecule of bone marrow stromal cell lines that facilitates pre-B-cell growth. Proc Natl Acad Sci USA 1994; 91: 5325-5329.

50 Hrycaj P, Korczowska I, Lacki JK: Severe Parkinson's disease in rheumatoid arthritis patient treated with infliximab. Rheumatology (Oxford) 2003; 42: 702-703.

51 Melikoglu MA, Sezer I, Kacar C: Rheumatoid-like hand deformities in Parkinson disease. J Clin Rheumatol 2007; 13: 236-237.

52 Kogure T, Tatsumi T, Kaneko Y, Okamoto K: Rheumatoid arthritis accompanied by Parkinson disease. J Clin Rheumatol 2008; 14: 192-193.

53 Emile J, Truelle JL, Pouplard A, Hurez D: Association of Parkinson's disease with HLA-B17 and B18 antigens. Nouv Presse Med 1977; 6: 4144

54 Elizan TS, Terasaki PI, Yahr MD: HLA-B14 antigen and postencephalitic Parkinson's disease. Their association in an American-Jewish ethnic group. Arch Neurol 1980; 37: 542-544.

55 Lampe JB, Gossrau G, Herting B et al: HLA typing and Parkinson's disease. Eur Neurol 2003; 50: 64-68.

56 Chen $\mathrm{H}$, Zhang SM, Hernan MA et al: Nonsteroidal anti-inflammatory drugs and the risk of Parkinson disease. Arch Neurol 2003; 60: 1059-1064.
57 Esposito E, Di Matteo V, Benigno A, Pierucci M, Crescimanno G, Di Giovanni G: Non-steroidal anti-inflammatory drugs in Parkinson's disease. Exp Neurol 2007; 205: 295-312.

58 McGeer PL, Schwab C, Parent A, Doudet D: Presence of reactive microglia in monkey substantia nigra years after 1-methyl-4-phenyl-1,2,3,6-tetrahydropyridine administration. Ann Neurol 2003; 54: 599-604.

59 Barcia C, Sanchez Bahillo A, Fernandez-Villalba E et al: Evidence of active microglia in substantia nigra pars compacta of parkinsonian monkeys 1 year after MPTP exposure. Glia 2004; 46: 402-409.

60 Gao HM, Hong JS, Zhang W, Liu B: Distinct role for microglia in rotenone-induced degeneration of dopaminergic neurons. J Neurosci 2002; 22: 782-790.

61 Lee DY, Oh YJ, Jin BK: Thrombin-activated microglia contribute to death of dopaminergic neurons in rat mesencephalic cultures: dual roles of mitogen-activated protein kinase signaling pathways. Glia 2005; 51: 98-110.

62 Wang XJ, Yan ZQ, Lu GQ, Stuart S, Chen SD: Parkinson disease IgG and C5a-induced synergistic dopaminergic neurotoxicity: role of microglia. Neurochem Int 2007; 50: 39-50.

63 Zhang W, Wang T, Pei Z et al: Aggregated alpha-synuclein activates microglia: a process leading to disease progression in Parkinson's disease. FASEB J 2005; 19: 533-542.

64 Grunblatt E, Mandel S, Jacob-Hirsch J et al: Gene expression profiling of parkinsonian substantia nigra pars compacta; alterations in ubiquitin-proteasome, heat shock protein, iron and oxidative stress regulated proteins, cell adhesion/cellular matrix and vesicle trafficking genes. J Neural Transm 2004; 111: 1543-1573.

65 Fung HC, Xiromerisiou G, Gibbs JR et al: Association of tau haplotype-tagging polymorphisms with Parkinson's disease in diverse ethnic Parkinson's disease cohorts. Neurodegener Dis 2006; 3: 327-333.

66 Vandrovcova J, Pittman AM, Malzer E et al: Association of MAPT haplotype-tagging SNPs with sporadic Parkinson's disease. Neurobiol Aging 2009; 30: 1477-1482.

Supplementary Information accompanies the paper on European Journal of Human Genetics website (http://www.nature.com/ejhg) 